\title{
MICROBIOLOGICAL CHARACTERISATION OF HAEMOPHILUS INFLUENZAE STRAINS ISOLATED FROM PATIENTS WITH INVASIVE AND RESPIRATORY DISEASES
}

\author{
Kostyanev T.*, L. Setchanova, V. Ouzounova-Raykova, Y. Proevska, I. Mitov \\ Department of Medical Microbiology, Faculty of Medicine, \\ Medical University - Sofia, Bulgaria
}

\section{SUMMARY}

A total of $175 \mathrm{H}$. influenzae strains were collected between 1994 and 2009 from all aged patient groups. The strains were isolated from patients with invasive and community-acquired respiratory tract infections. All strains were identified according to standard microbiological methods. Serotyping was done by a coagglutination test and by molecular PCR capsular genotyping. Beta-lactamase production was determined by the chromogenic cephalosporin test with nitrocephin as substrate.

Most of the isolated $H$. influenzae strains were from children under 5 years of age (57.7\%). Overall, 61 strains belonged to serotype $b(34.9 \%)$ by the means of PCR capsular typing, 1 strain was type $\mathrm{f}$, and 113 isolates (64.6\%) were non-typeable (non-encapsulated) H. influenzae. Among the infants and children with meningitis or other invasive infections, aged 2 month to 5 years, all strains, except one, were serotype b. In respiratory tract infections (pneumonia, otitis media, sinusitis and people with chronic pulmonary diseases - exacerbations of COPD, bronchiectasis, cystic fibrosis) the most common - $96.5 \%$ were non-typeable strains in both groups children and adults. Overall, the prevalence of beta-lactamase production was $19.4 \%$. But, it was much higher for invasive strains from CSF isolates $37.7 \%, 25 \%$ in blood samples, and $37.5 \%$ in otitis media causative strains. Beta-lactamase production was less frequent in respiratory tract isolates - in sputum $13.3 \%$ and in URT samples - $2.3 \%$. The rate of beta-lactamase production in CSF isolates has not changed for the last 10 years.

PCR capsular genotyping method has to be performed for all non-b-type strains. The implementation of Hib vaccine in our country will be accompanied by a reduction in invasive diseases caused by $H$. influenzae type $\mathrm{b}$ in children, but it is not useful in preventing infections caused by non-typeable $H$. influenzae strains.

Key words: $H$. influenzae, capsular genotyping, nontypeable $H$. influenzae, beta-lactamase

\section{INTRODUCTION}

Haemophilus influenzae is one of the leading bacterial agents, causing community-acquired invasive and respiratory diseases in both children and adults.

New $H$. influenzae type b conjugate vaccine (Hib) is now available. Bulgarian Ministry of Health decided to introduce vaccination against Hib disease in our childhood vaccination schedule starting in April 2010.

The aims of this study were:

1. To determine different capsular types by PCR genotyping of $\mathrm{H}$. influenzae strains isolated from children and adults with invasive and respiratory diseases before the introduction of Hib conjugate vaccine in our country.

2. To study the microbiological features of the collected isolates: beta-lactamase production; biotypes and other clinical parameters.

\section{MATERIALS AND METHODS}

Bacterial isolates. A total of $175 \mathrm{H}$. influenzae strains were collected between 1994 and 2009 from all aged groups. They were isolated from cerebrospinal fluid (CSF) $(n=53)$, blood $(n=8)$, sputum $(n=60)$, ear fluid $(n=8)$ and upper respiratory tract (URT) $(n=46)$. Patients from whom the strains were isolated had meningitis, septicemia or community-acquired respiratory tract infections (otitis media in children, pneumonia, bronchitis, conjunctivitis or acute exacerbations of chronic obstructive pulmonary disease in adults).

All strains were identified according to the standard microbiological methods [4]. Beta-lactamase production was determined by the chromogenic cephalosporin test with nitrocephin as substrate (Cefinase, BBL, BD).

Serotyping of H.influenzae. The strains were serotyped by a coagglutination test (Phadebact Haemophilus test; Bactus AB, Huddinge, Sweden) and by molecular PCR capsular typing method.

DNA isolation. DNA isolation was performed from a 24-hour culture on chocolate agar containing 1\% isovitalex. One loopful of each bacterial culture was suspended in 400 $\mu 1$ of sterile distilled water and then boiled for $3 \mathrm{~min}$. The 
boiling extract was centrifuged at $12,000 \mathrm{x}$ g for $10 \mathrm{~min}$. The supernatant was then collected.

PCR of capsular genes. The genes were amplified with primer sets by Falla et al. [2] and listed in Table 1. PCR was implemented by using $2.5 \mu \mathrm{l}$ of template DNA, $1 \mu \mathrm{M}$ of each primer, $0.2 \mathrm{mM}$ deoxyribonucleotides, $1 \mathrm{x}$ reaction buffer, 2 $\mathrm{mM} \mathrm{MgCl} 2$ and $0,5 \mathrm{U}$ of Taq polymerase in a total volume of $25 \mu \mathrm{l}$. The DNA was amplified using the following protocol: initial denaturation $\left(94^{\circ} \mathrm{C}\right.$ for $\left.5 \mathrm{~min}\right)$, followed by 25 cycles of denaturation $\left(94^{\circ} \mathrm{C}\right.$ for $\left.1 \mathrm{~min}\right)$, annealing $\left(60^{\circ} \mathrm{C}\right.$ for $\left.1 \mathrm{~min}\right)$, and extension $\left(72^{\circ} \mathrm{C}\right.$ for $\left.1 \mathrm{~min}\right)$. After the final cycle there was a single final extension of $10 \mathrm{~min}$ at $72^{\circ} \mathrm{C} .10 \mu \mathrm{l}$ of PCR products were separated for $30 \mathrm{~min}$ at $130 \mathrm{~V}$ in $1 \%$ agarose in Tris-Clborate-EDTA buffer and visualized by ethidium bromide staining $(0.5 \mathrm{Mg} / \mathrm{ml})$. The size of the amplified products was compared with a positive control, obtained from CDC, Atlanta, USA. The detection was performed by UV transillumination (wavelength $312 \mathrm{~nm}$ ).

Table 1. Capsule type-specific PCR primers

\begin{tabular}{ccl}
\hline a to f & HI-1 & CGT TTG TAT GAT GTT GAT CCA GAC \\
& HI-2 & TGT CCA TGT CTT CAA AAT GAT G \\
a & a1 & CTA CTC ATT GCA GCA TTT GC \\
& a2 & GAA TAT GAC CTG ATC TTC TG \\
b & b1 & GCG AAA GTG AAC TCT TAT CTC TC \\
& b2 & GCT TAC GCT TCT ATC TCG GTG AA \\
c & c1 & TCT GTG TAG ATG ATG GTT CA \\
& c2 & CAG AGG CAA GCT ATT AGT GA \\
d & dl & TGA TGA CCG ATA CAA CCT GT \\
& d2 & TCC ACT CTT CAA ACC ATT CT \\
e & e1 & GGT AAC GAA TGT AGT GGT AG \\
& e2 & GCT TTA CTG TAT AAG TCT AG \\
f & fl & GCT ACT ATC AAG TCC AAA TC \\
& 2 2 & CGC AAT TAT GGA AGA AAG CT \\
\hline
\end{tabular}

\section{RESULTS AND DISCUSSION}

Taking in consideration the specimens' source of isolates and the patients' age, it was obvious that the meningitis and other invasive diseases were the most common (91.8\%) in children less than 5 years of age (56 out of 61 cases) and they were caused predominantly by serotype $\mathrm{b}$ of $H$. influenzae (95.1\%).

Alternatively, in respiratory tract infections (RTI-114 cases), $39.5 \%$ of the isolates were from children under the age of 5 years and $48.2 \%$ were from adult patients. (Fig. 1)

Serotype PCR determination was performed in two steps and it characterized all $175 \mathrm{H}$. influenzae strains as follows: $61(34.9 \%)$ strains were type b, $1(0.6 \%)$ isolate type f, and the rest 113 (64.6\%) strains were non-capsulated or non- typeable H. influenzae (NTHi). (Fig. 2) The first set of primers (HI1 and HI2) amplified a 343 bp product only from the 62 capsulate strains (Fig. 3). All the others, previously serotyped by the coagglutination test, were negative for the capsular expression gene (bexA) and hence, non-capsulated. The second set of primers (b1 and b2) used, revealed a $480 \mathrm{bp}$ product from all the capsulate strains but one (Fig. 4). They were clearly confirmed as $H$. influenzae type $\mathrm{b}$, possessing duplicated capB locus. The second primers' set was also used to verify whether all NC strains are really lacking capsular genes or amongst these there are so called mutant bII strains. These strains are indistinguishable from NC strains by traditional serotyping techniques but can be determined by the PCR method. As a result of losing one of the copies of the cap gene and the bexA gene they fail to produce and express a capsule. They can be detected after amplification with $\mathrm{b} 1$ and $\mathrm{b} 2$ primers, producing type $\mathrm{b}$-specific product. All the 113 strains which failed to produce product with HI-1 and HI-2, were also negative with b1 and b2 which excluded the presence of capsule-deficient mutants among the isolates.

There was a total match between the coagglutination test and the PCR method for serotyping of $H$. influenzae. But PCR capsular genotyping was more useful to distinguish different capsular serotypes (one of type f) and capsuledeficient mutants of type b strains - b- strains (none was found in this study).

Serotype distribution in patients with RTI from all age groups showed that NTHi strains were the most commonly found isolates - $96.5 \%$. Only two strains isolated from mucosal respiratory infections were type $\mathrm{b}(2.6 \%)$ and 1 was type $\mathrm{f}(0.9 \%)$. (Fig. 5$)$

Overall, beta-lactamase production was detected in $19.4 \%$ of the 175 tested $H$. influenzae strains. в-lactamase production was higher in children with meningitis (37.7\%) and otitis media (37.5\%), but it was less in mucosal respiratory isolates $(2.3 \%)$ and sputum $(13.3 \%)$ in both age groups children and adults. (Fig. 6)

Beta-lactamase production in Hib strains has not changed over the last 15 years in children with meningitis [3].

Fig. 1. Distribution of H. influenzae isolates (n) among different age groups.

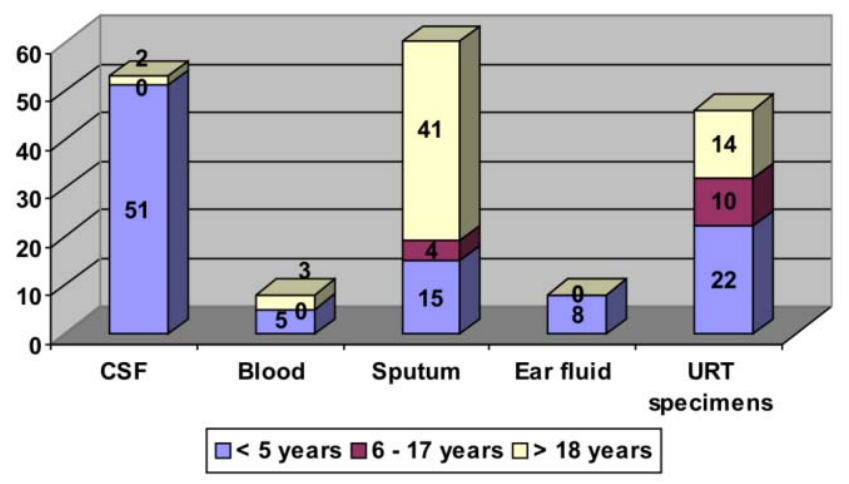


Fig. 2. H. influenzae serotype distribution (n) from different specimens.

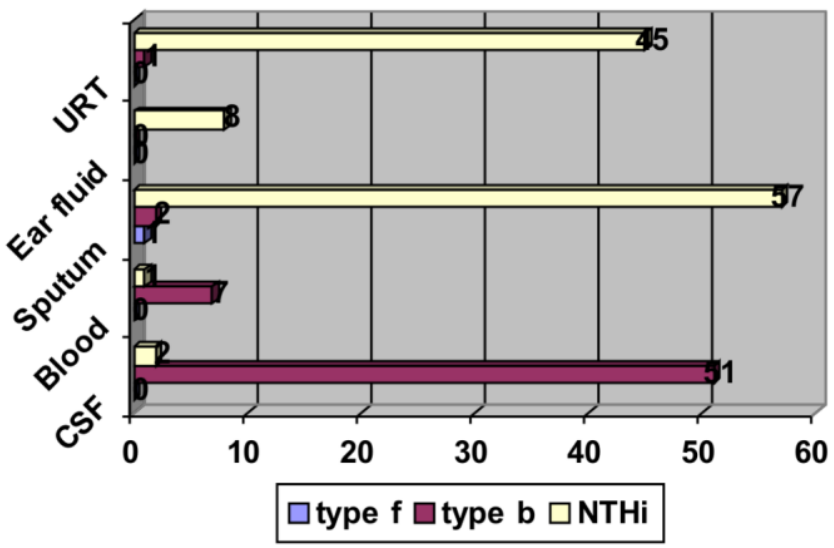

Fig. 3. Gel electrophoresis of PCR products (343 bp) for the detection of bexA gene of $H$. influenzae:

Lane $\mathrm{M}-100$ bp Ladder; Lane 1 - positive control; Lane 2 - Negative strain; Lane 3 - Positive isolate from CSF; Lane 4 - Positive isolate from blood; Lane 5 - Positive isolate from sputum; Lane 6 - Positive isolate from nasopharyngeal swab; Lane 7 - negative control

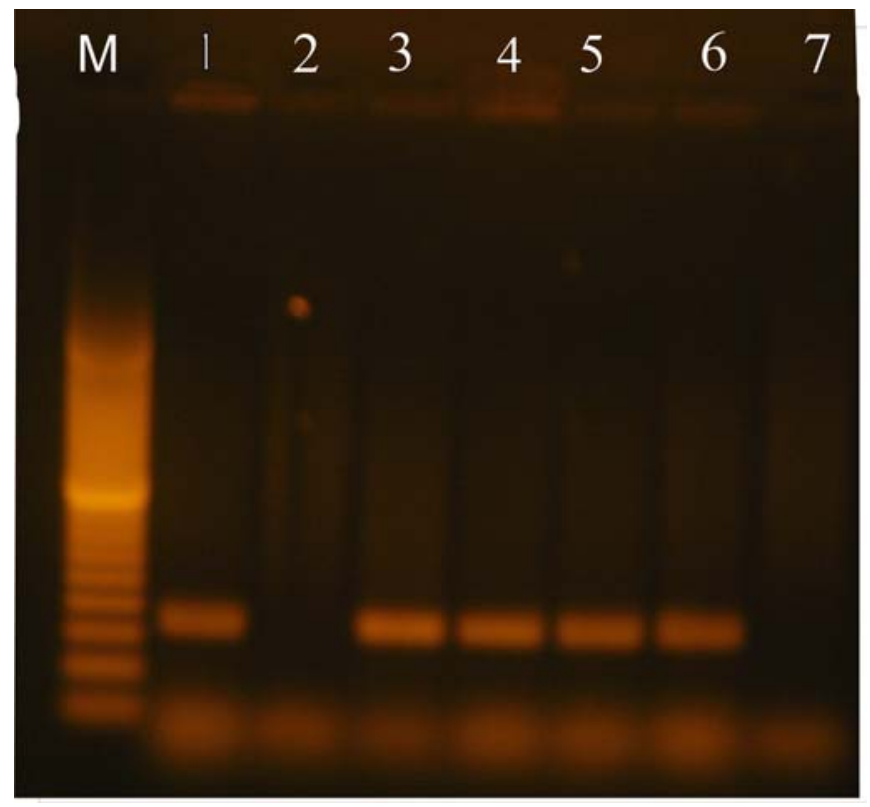

Fig. 4. Gel electrophoresis of PCR products (480 bp) for the detection of capsular type b of $H$. influenzae:

Lane M - 100 bp Ladder; Lane 1 - positive control; Lane 2 - Negative strain; Lane 3 - Positive isolate from CSF; Lane 4 - Positive isolate from blood; Lane 5 - Positive isolate from sputum; Lane 6 - Positive isolate from nasopharyngeal swab

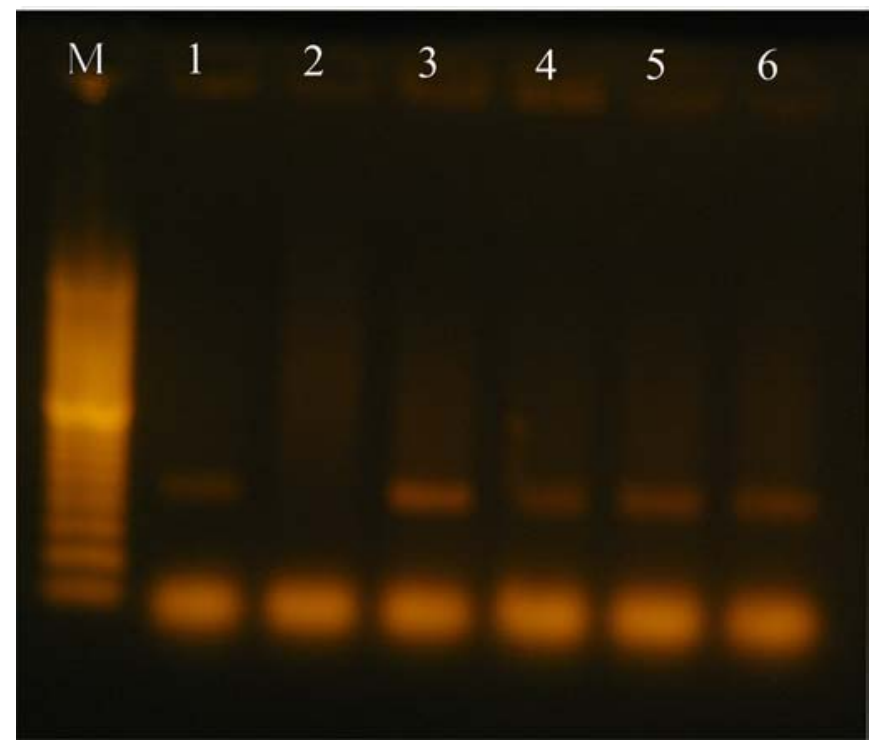

Fig. 5. Gel electrophoresis of PCR products (450 bp) for the detection of capsular type $\mathrm{f}$ of $H$. influenzae:

Lane M - 100 bp Ladder; Lane 1 - positive control; Lane 2 - Negative strain; Lane 3 - Positive isolate from sputum

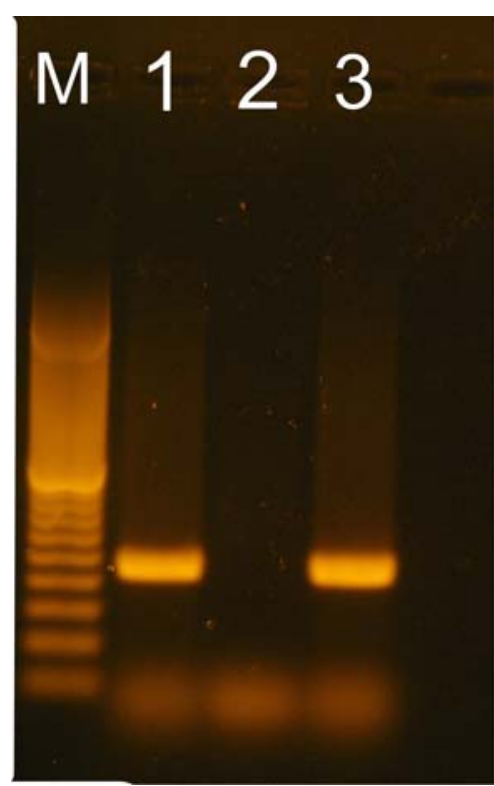


Fig. 6. Frequency of beta-lactamase producing $H$. influenzae isolates.

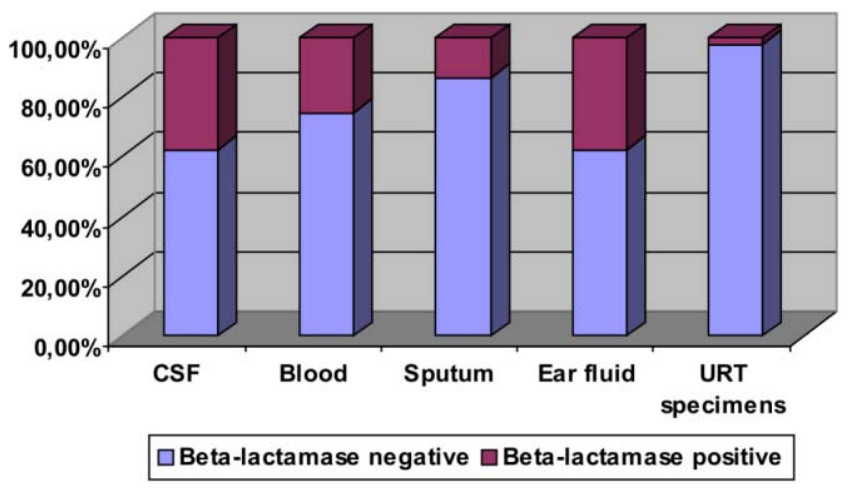

Acknowledgments:

This study was supported by research grant from the Medical University of Sofia, Bulgaria (Project No.16, Contract No.18/2009).

\section{REFERENCES:}

1. Murphy, T.F. 2003. Respiratory infections caused by non-typeable Haemophilus influenzae. Curr Opin Infect Dis 16: 129-134.

2. Falla T.J., D. W. Crook, L.N. Brophy et al. 1994. PCR for Capsular Typing of

\section{CONCLUSIONS}

1. H. influenzae type $\mathrm{b}$ is the most common serotype among the infants and children with meningitis or other invasive infections, aged 2 months to 5 years. Approximately one-third of the Hib isolates were beta-lactamase producing strains.

2. The introduction of Hib vaccine in our National Vaccination Plan will be accompanied by reduction in both the incidence of Hib invasive disease in children and the incidence of beta-lactamase producing Hib strains.

3. Non-typeable $H$. influenzae are more common than Hib in respiratory tract infections in both children and adults. Hib vaccine is not useful in preventing infections caused by non-typeable $H$. influenzae (NTHi) strains.

4. There is a need for continued microbiological and epidemiological surveillance on serotype distribution of $H$. influenzae and surveillance to collect data on clinical presentations of NTHi to emphasize the importance of immunization against this disease.
Haemophilus influenzae. J.Clin. Microbiol. 32:2382-2386.

3. Сечанова Л. Микробиологичен подход в етиологичната терапия на острия бактериален менингит. Инфекто- логия. 2002. XXXIX, 2: 25-28.

4. Kilian M. Haemophilus. 2007. In: Manual of Clinical Microbiology (9th ed.) ed by Murray P.R. et al. ASM press, Washington, DC, USA.

Address for correspondence:

Dr. Tomislav Kostyanev, MD

Department of Medical Microbiology, Medical University - Sofia

2, Zdrave Str., 1431 Sofia, Bulgaria

Tel.: +35929172 722; Fax: +35929515317

E-mail: t_kostyanev@yahoo.co.uk 